\title{
In vitro human embryonic stem cell hematopoiesis mimics MYB-independent yolk sac hematopoiesis
}

\author{
Stijn Vanhee, ${ }^{1}$ Katrien De Mulder, ${ }^{1}$ Yasmine Van Caeneghem, ${ }^{1}$ Greet Verstichel, ${ }^{1}$ Nadine Van Roy, ${ }^{2}$ Björn Menten, ${ }^{2}$ \\ Imke Velghe, ${ }^{1}$ Jan Philippé, ${ }^{3}$ Dominique De Bleser, ${ }^{4}$ Bart N. Lambrecht,,${ }^{5,6}$ Tom Taghon, ${ }^{1}$ Georges Leclercq, ${ }^{1}$ Tessa Kerre ${ }^{1}$ \\ and Bart Vandekerckhove ${ }^{1}$
}

${ }^{1}$ Laboratory for Experimental Immunology, Ghent University; ${ }^{2}$ Center for Medical Genetics, Ghent University; ${ }^{3}$ Department of Clinical Biology, Microbiology and Immunology, Ghent University Hospital; ${ }^{4}$ Red Cross Flanders, Ghent; ${ }^{5}$ Laboratory of Immunoregulation and Mucosal Immunology, Department of Pulmonary Medicine, Ghent University Hospital; and ${ }^{6}$ Flanders Institute for Biotechnology (VIB) Inflammation Research Center, Ghent University, Belgium

\section{ABSTRACT}

Although hematopoietic precursor activity can be generated in vitro from human embryonic stem cells, there is no solid evidence for the appearance of multipotent, self-renewing and transplantable hematopoietic stem cells. This could be due to short half-life of hematopoietic stem cells in culture or, alternatively, human embryonic stem cellinitiated hematopoiesis may be hematopoietic stem cell-independent, similar to yolk sac hematopoiesis, generating multipotent progenitors with limited expansion capacity. Since a MYB was reported to be an excellent marker for hematopoietic stem cell-dependent hematopoiesis, we generated a MYB-eGFP reporter human embryonic stem cell line to study formation of hematopoietic progenitor cells in vitro. We found CD34 ${ }^{+}$hemogenic endothelial cells rounding up and developing into $\mathrm{CD}^{4} 3^{+}$hematopoietic cells without expression of MYB-eGFP. MYB-eGFP+ cells appeared relatively late in embryoid body cultures as $\mathrm{CD} 34^{+} \mathrm{CD} 43^{+} \mathrm{CD} 45^{-10}$ cells. These $\mathrm{MYB}-\mathrm{eGFP}{ }^{+}$cells were CD33 positive, proliferated in IL-3 containing media and hematopoietic differentiation was restricted to the granulocytic lineage. In agreement with data obtained on murine $M y b^{-1-}$ embryonic stem cells, bright eGFP expression was observed in a subpopulation of cells, during directed myeloid differentiation, which again belonged to the granulocytic lineage. In contrast, $\mathrm{CD}_{14}{ }^{+}$macrophage cells were consistently eGFP- ${ }^{-}$and were derived from eGFPprecursors only. In summary, no evidence was obtained for in vitro generation of $\mathrm{MYB}^{+}$hematopoietic stem cells during embryoid body cultures. The observed $M Y B$ expression appeared late in culture and was confined to the granulocytic lineage.

\section{Introduction}

During embryogenesis, hematopoietic development is spatiotemporally organized in different waves. During the first, so-called 'primitive' wave of hematopoiesis, nucleated erythrocytes and macrophage-like cells are generated in the yolk sac (YS). Subsequent waves generate multipotent progenitor cells, first in the YS and, finally, in the aorta-gonadomesonephros region (AGM) where hematopoietic stem cells (HSCs) are generated.

Members of the MYB transcription factor family are important transcriptional regulators throughout embryonic development. One of these three family members, MYB, is differentially expressed during embryonic hematopoietic development. ${ }^{2}$ While $M y b$ transcripts have been detected at low levels during early waves of hematopoiesis, this process is not MYB-dependent. In contrast, HSC-dependent hematopoiesis appears to strictly rely on MYB as loss of function mutants of MYB lead to embryonic lethality due to failure of fetal liver hematopoiesis. ${ }^{3,4}$ In the zebrafish, transgenic animals expressing eGFP under control of myb regulatory elements have been used for visualization and analysis of HSC generation from aortic blood forming hemogenic endothelium. ${ }^{5}$

In postnatal life, long-term repopulating hematopoietic stem cells (LT-HSC) were found to express the highest levels of $M y b$ and levels decrease progressively in the short-term repopulating HSC (ST-HSC) and in the multipotent progenitor population (MPP). ${ }^{6,7}$

MYB-independent hematopoiesis consists largely of shortlived precursors and mature blood cells. However, the mononuclear phagocyte system (MPS), consisting of brain microglial cells, hepatic Kupffer cells and skin Langerhans cells, seems to be derived from MYB-independent hematopoietic progenitor cells (HPC) rather than bone marrow-derived MYB-dependent HSC. Recently, Schulz and colleagues have shown that two parallel pathways of macrophage differentiation can be distinguished by their inherent dependence on MYB. ${ }^{8} \mathrm{MYB}$-independent cells originate in the YS around embryonic day 7.5-8.5 (E7.5-E8.5), possibly from an erythro-myeloid restricted precursor (EMP), while MYB-dependent macrophages originate from bone marrow HSC.

Gene therapeutic strategies for inherited immune deficiencies or other genetic diseases of the blood rely on the assumption that HSC can be generated in vitro from pluripotent stem cells. ${ }^{9}$ In these approaches, patient-specific induced pluripotent stem cells are generated and the defective gene is corrected by homologous recombination subsequent to targeting the gene defect by zinc-finger nucleases (ZFN) or TALENs. Once the genetic defect is corrected, 'cured' HSC are generated by

(C)2015 Ferrata Storti Foundation. This is an open-access paper. doi:10.3324/haematol.2014.112144

The online version of this article has a Supplementary Appendix.

Manuscript received on June 11, 2014. Manuscript accepted on November 4, 2014.

Correspondence: bart.vandekerckhove@ugent.be 
A

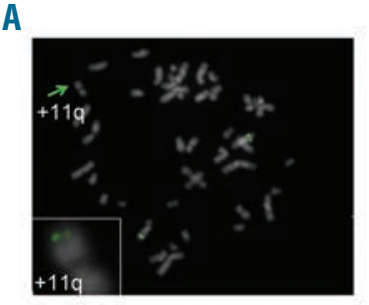

c

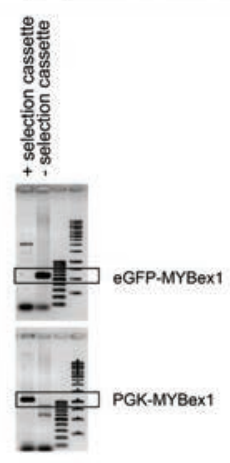

D
B
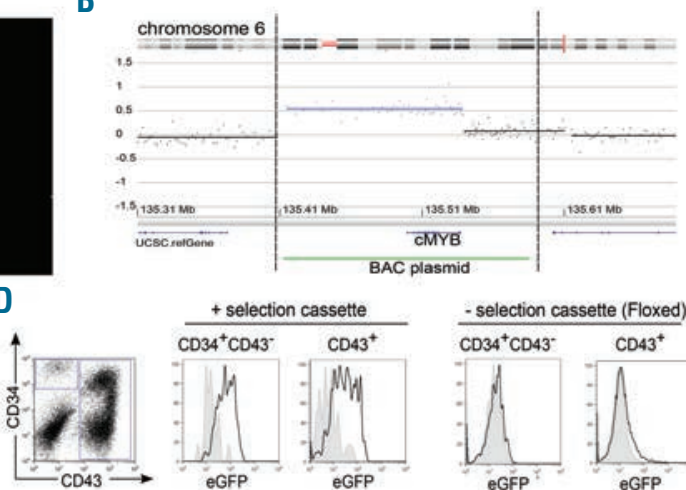
$\mathrm{CD}^{+} 4^{+} \mathrm{CD}^{-} 3^{-}$

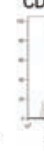
E MYB-eGFP

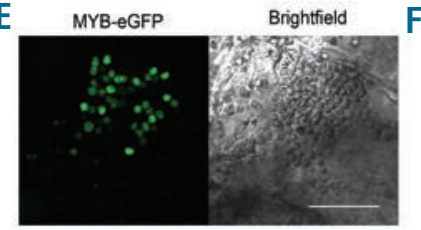

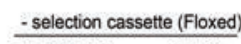
$\mathrm{CD} 34^{+} \mathrm{CD} 43^{-}$

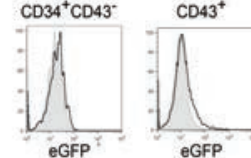

$F$

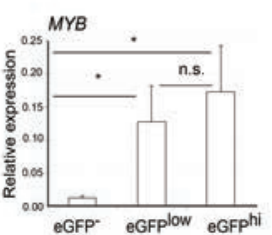

Figure 1. Functional validation of MYB-eGFP reporter hESC. (A) MYB FISH analysis (green) on metaphase nuclei (DAPI stain in gray); inset shows chromosome 11 only. (B) ArrayCGH showing amplification of the genomic region within the BAC plasmid. A relevant portion of chromosome 6 is shown. (C) Validation of selection cassette removal, as assessed by PCR ranging from eGFP to the first exon of MYB (upper gel) or ranging from pPGK to the first exon of MYB (lower gel). (D) MYB-eGFP reporter hESCs differentiated towards the hematopoietic lineage were analyzed for eGFP expression. $\mathrm{CD}_{34}{ }^{+} \mathrm{CD} 43^{-}$endothelial and $\mathrm{CD} 43^{+}$hematopoietic populations are depicted. Both floxed and non-floxed cell lines are shown. (E) Confocal fluorescence microscopy of a day 20 embryoid bodies (EB) culture in EB mix showing bright eGFP positive round cells. Scale bar measures $100 \mu \mathrm{M}$. (F) MYB qPCR analysis of eGFP sorted hematopoietic cells from EB differentiation culture. Expression is shown relative to the mean of GAPDH and YWHAZ expression. Error bars indicate standard deviation (SD) of the mean $(n=4)$. an appropriate in vitro differentiation protocol before infusion. However, current protocols have failed to convincingly demonstrate the generation of HSC in pluripotent stem cell differentiation cultures.

To investigate whether during in vitro human embryonic stem cell (hESC)-derived hematopoiesis HSC are formed or rather hematopoiesis from hESC depends on the emergence of a MYB independent EMP-like cell, we generated a $M Y B$ reporter line using random integration of a bacterial artificial chromosome (BAC) reporter construct in which eGFP expression is under control of the MYB regulatory DNA sequences.

\section{Methods}

\section{Cell lines, culture of cell lines and isolation of primary cells}

All experiments were approved by the Medical Ethical Committee of Ghent University Hospital, Belgium. The WA01 (National Institutes of Health code: WA01) human embryonic stem cell (hESC) line was used in all experiments. A detailed description of the methods used for cells and cell cultures is available in the Online Supplementary Appendix.

\section{Hematopoietic differentiation of hESC in spin embryoid bodies}

To differentiate hESC into hematopoietic cells, the protocol from $\mathrm{Ng}$ et al. was used with minor modifications. ${ }^{10}$ In brief, $5 \times 10^{3}$ single cell-adapted hESC were spun at $480 \mathrm{~g}$ into each well of $96-$ well low attachment plates and subsequently cultured in APEL medium containing $10 \mu \mathrm{M}$ Rock inhibitor Y-27632 (Selleckchem, Houston, TX, USA), 40 ng/mL SCF (Peprotech, Rocky Hill, NJ, USA), $2 \mathrm{ng} / \mathrm{mL}$ BMP4 (R\&D, Minneapolis, MN, USA) and 20 $\mathrm{ng} / \mathrm{mL}$ VEGF165 (Peprotech), further referred to as "embryoid body (EB) mix". After four days, spin EB were transferred onto an OP9 cell layer and further cultured in EB mix for a total of 7-14 days. Half of the medium was changed on day 7 (d7), with APEL medium containing EB mix cytokines, unless a different combination of cytokines is specified. These cytokines were added at the following concentrations: $50 \mathrm{ng} / \mathrm{mL}$ IL-3 (R\&D), $50 \mathrm{ng} / \mathrm{mL}$ Flt3-L (R\&D), 10 ng/mL TPO (Peprotech) and/or 50 ng/mL IL-6 (R\&D).
For myeloid differentiation, spin EBs were dissociated at d11 and transferred onto OP9 cells in MEM-a with $20 \%$ FCS, supplemented with $50 \mathrm{ng} / \mathrm{mL}$ SCF (Peprotech), $50 \mathrm{ng} / \mathrm{mL}$ TPO (Peprotech), 10 ng/mL IL-3 (R\&D) and 20 ng/mL IL-6 (R\&D). For erythro-megakaryocytic differentiation, spin EBs were dissociated and transferred onto OP9 cells in MEM-a with $20 \%$ FCS, supplemented with $50 \mathrm{ng} / \mathrm{mL}$ SCF (Peprotech), $50 \mathrm{ng} / \mathrm{mL}$ TPO (Peprotech) and $50 \mathrm{ng} / \mathrm{mL}$ EPO (eBioscience, San Diego, CA, USA).

\section{Flow cytometry and cell sorting}

Flow cytometric analysis was performed on an LSR II system (BD Biosciences, San Jose, CA, USA). Cell sorting was performed with a FACS ARIA IIIU system (BD Biosciences). A list of the antibodies used can be found in the Online Supplementary Appendix.

\section{Real-time RT-qPCR}

Cells were lysed and cDNA was synthesized using the SYBR power cells-to-Ct system (Ambion, Life Technologies, Carlsbad, CA, USA) according to the manufacturer's instructions. Primer sequences can be found in Online Supplementary Table S1. PCR reagents and SYBR GreenI master were obtained from Roche (Roche, Penzberg, Germany) and used according to the manufacturer's instructions. The reactions were run on a lightcycler480 384-well system (Roche).

\section{Statistical analysis}

All statistical analyses were performed using SPSS v.22.0 (IBM, New York, NY, USA). Significance was assessed using MannWhitney U statistical analysis; $P \leq 0.05$ was considered significant.

Additional information on materials and methods is provided in the Online Supplementary Appendix.

\section{Results}

\section{Generation of MYB-eGFP transgenic reporter hESC}

To study MYB expression during hematopoietic differentiation, we generated a MYB-eGFP reporter hESC line using random insertion bacterial artificial chromosome 
A

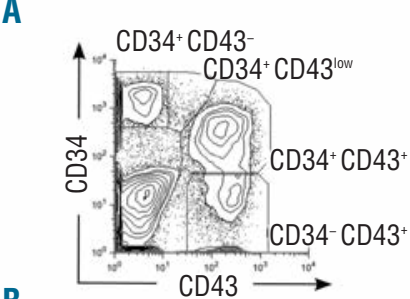

B
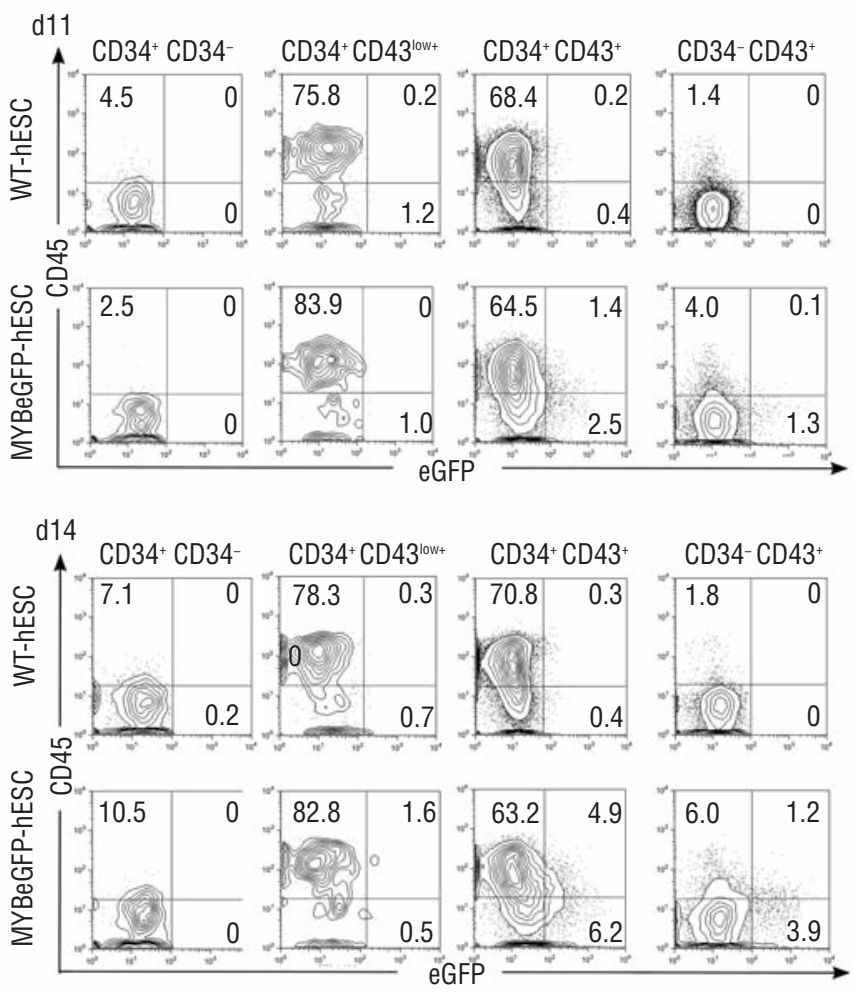

Figure 2. MYB-eGFP ${ }^{+}$HPC are generated relatively late in embryoid bodies cultures. (A) Gating strategy for assessment of eGFP expression, a representative plot of embryoid bodies (EB) day 11 (d11) culture is shown. (B) Flow cytometric analysis of d11 and d14 EB cultures in EB mix. Wildtype $\mathrm{hESC}$ are presented as control for eGFP signal in all populations. Percentages of cells in each quadrant are shown.

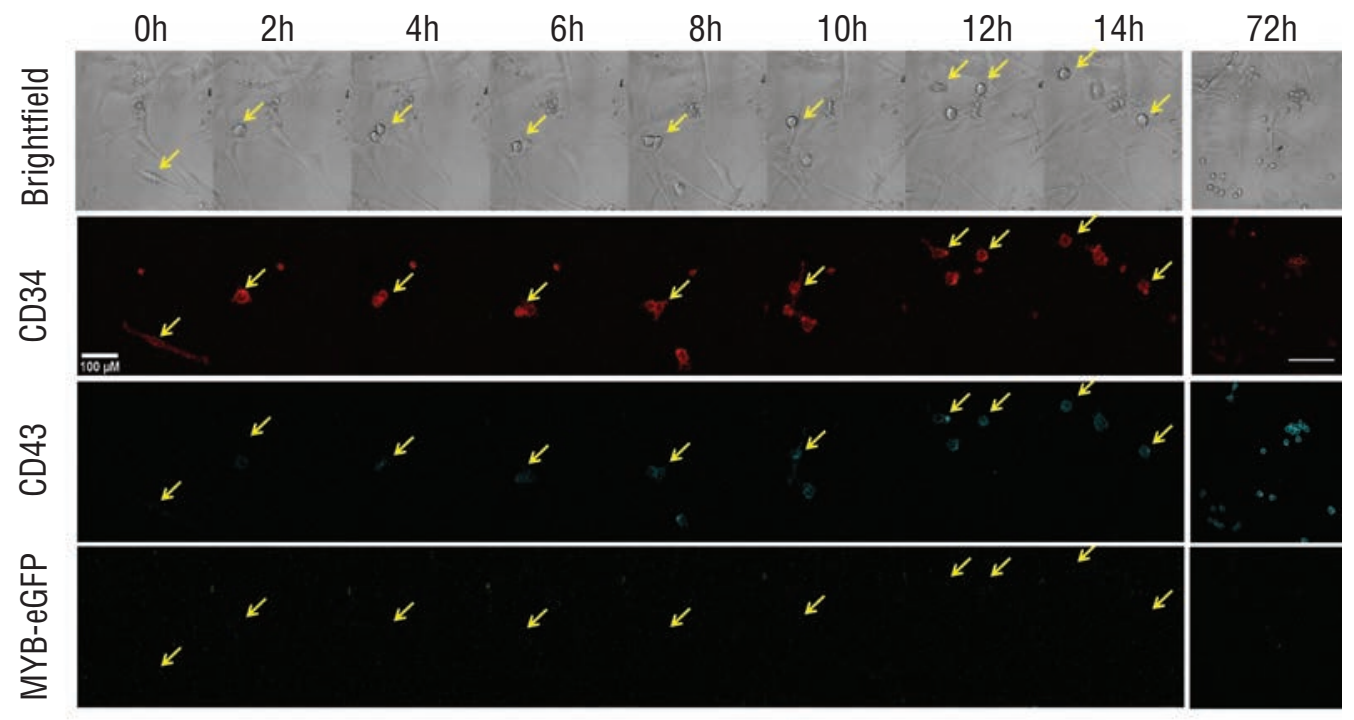

Figure 3. Hematopoietic cells are generated directly from hemogenic endothelium, without an eGFP+ intermediate. Time-lapse confocal analysis of re-plated day 11 (d11) hemogenic endothelium. A single frame is depicted every $2 \mathrm{~h}$ starting from the defined starting point. Cropped images are shown for every channel. Arrows depict a single endothelial cell at the starting point of analysis and the progeny thereof at later points in time. Scale bar measures $100 \mu \mathrm{M}$. At $72 \mathrm{~h}$, the cells have rapidly proliferated and generated two clusters of 8 cells. These cells have down-regulated CD34 and up-regulated CD43. Scale bar measures $100 \mu \mathrm{M}$. 
A

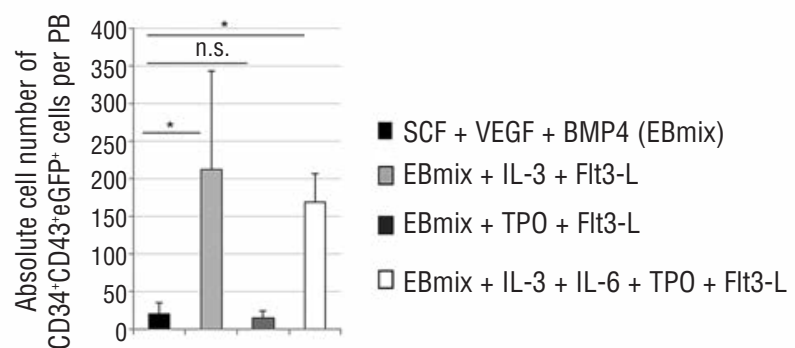

B

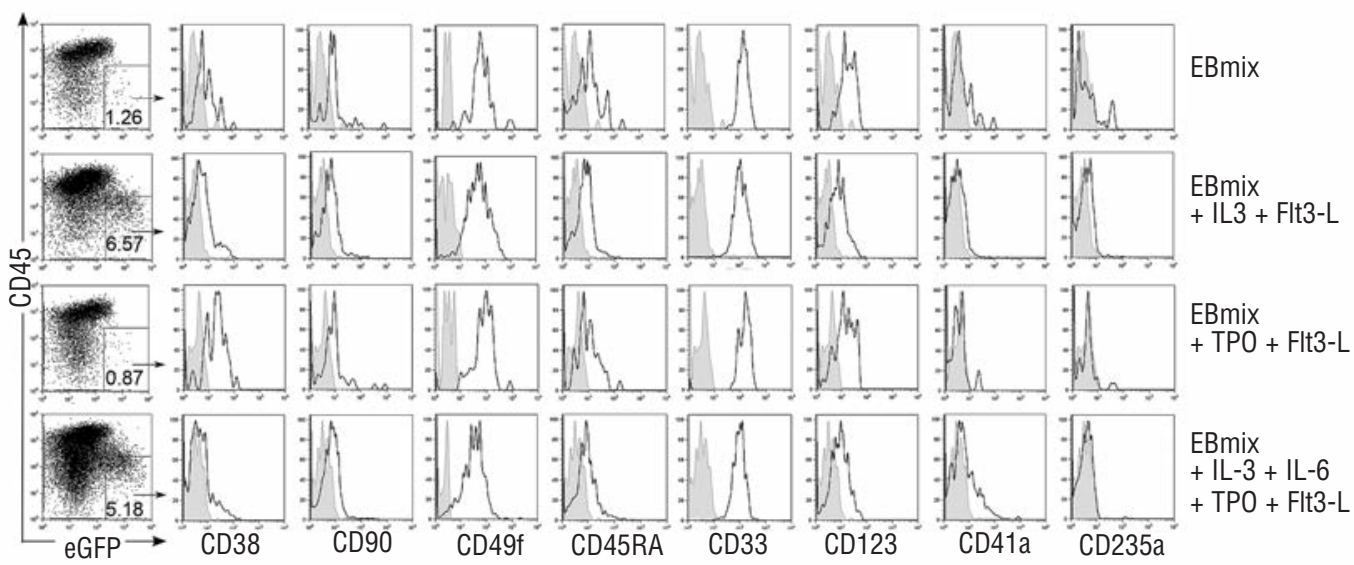

Figure 4. Characterization of MYB-eGFP+ ${ }^{+}$PPC. (A) Absolute cell number of eGFP cells within the $\mathrm{CD}^{+} 4^{+} \mathrm{CD} 43^{+}$population of day 14 (d14) $^{-}$ embryoid bodies (EB) cultures. Error bars indicate standard deviation (SD) of the mean ( $n=3$ ). (B) Flow cytometric analysis of d14 MYB-eGFP $\mathrm{EB}$, cultured in the presence of different cytokine mixes as indicated. Dot plots are gated on CD34 ${ }^{+} \mathrm{CD} 43^{+}$. Cells within the eGFP ${ }^{+}$gate are depicted in the histograms. Shaded histograms show isotype control stained samples for the indicated marker. Representative plots of 3 independent experiments are shown.

(BAC) transgenesis (Online Supplementary Figure S1).

After nucleofection of hESC and neomycin selection, neomycin-resistant hESC colonies were obtained. Figure 1 shows data for clone 5, which gave the highest eGFP expression and was used in all experiments. Besides the endogenous $M Y B$ loci on chromosome 6q, a clear hybridization signal on chromosome 11q was detected on FISH analysis, confirming successful integration of the BAC reporter vector (Figure 1A). High-resolution arrayCGH shows integration of the BAC plasmid from +84.6 kbp 5' of the MYB start codon to $-39.3 \mathrm{kbp} 3$ ' of the start codon. This region encompasses the complete MYB gene and upstream regulatory elements (Figure $1 \mathrm{~B}$ ).

Hematopoietic differentiation of the MYB-eGFP cell line, before removal of the selection cassette, showed eGFP expression by $\mathrm{CD}_{4} 3^{+}$hematopoietic cells as well as by $\mathrm{CD} 34^{+} \mathrm{CD} 43^{-}$endothelial cells (Figure 1D). As no $M Y B$ transcripts could be found by GPCR analysis in the $\mathrm{CD} 34^{+} \mathrm{CD} 43^{-}$population (Online Supplementary Figure S2), aberrant expression due to interference of the pGK promotor was hypothesized. Therefore, the pGK promotordriven selection cassette was removed through transient transfection with a Cre recombinase-encoding plasmid (Figure 1C). After removal of the selection cassette, no eGFP expression was observed in non-hematopoietic cells (Figure 1D). Although the expression of eGFP found at this point in time was relatively weak, prolonged culture clearly showed strong eGFP ${ }^{+}$cells as determined by confocal microscopy analysis of $\mathrm{d} 20 \mathrm{~EB}$ differentiation cultures (Figure 1E).

Fidelity of the random integration reporter cell line was validated by qPCR analysis for MYB expression. eGFP-, eGFPlow and eGFPhi cells were sorted from d14 hematopoietic differentiation cultures in the presence of SCF, TPO, IL-3 and IL-6 and MYB mRNA levels were shown to correlate with eGFP protein expression in the sorted populations (Figure $1 \mathrm{~F}$ and Online Supplementary Figure S2). Note that the eGFP- cells are not completely negative for $M Y B$ mRNA. This indicates that the reporter line is not as sensitive as RT-qPCR for MYB expression. However, this does not preclude the detection of HSC, as these cells are supposed to express high levels of $M y b{ }^{6}$

\section{Endothelium-derived early hematopoietic precursors are MYB-eGFP negative}

Spin EB cultures were set up and screened daily for the appearance of hematopoietic eGFP ${ }^{+}$cells. Expression of CD43, the earliest marker for hematopoietic cells, was first observed in our cultures at $d 7$. Few eGFP+ cells became apparent from d11 onwards, forming a clear population on d14. As shown in Figure $2 \mathrm{~A}$ and $\mathrm{B}$, these eGFP $\mathrm{GF}^{+}$ cells were contained within the $\mathrm{CD} 34^{+} \mathrm{CD} 43^{+}$population, which has been reported to contain progenitors of multiple hematopoietic lineages. On the other hand, the CD34+ CD $43^{-}$endothelial and $\mathrm{CD} 34^{+} \mathrm{CD} 43^{\text {lo }}$ emerging HPC populations were consistently negative for eGFP expression (Figure 2B). As expression was also found highest in the $\mathrm{CD} 34^{+} \mathrm{CD} 43^{+}$population within the $\mathrm{CD} 45^{\text {low }}$ population (Online Supplementary Figure S2), we hypothesized this population to have the highest possibility of containing $\mathrm{MYB}^{+}$multipotent progenitors.

To assess whether the eGFP ${ }^{+} \mathrm{CD} 3^{+}$hematopoietic cells 
A

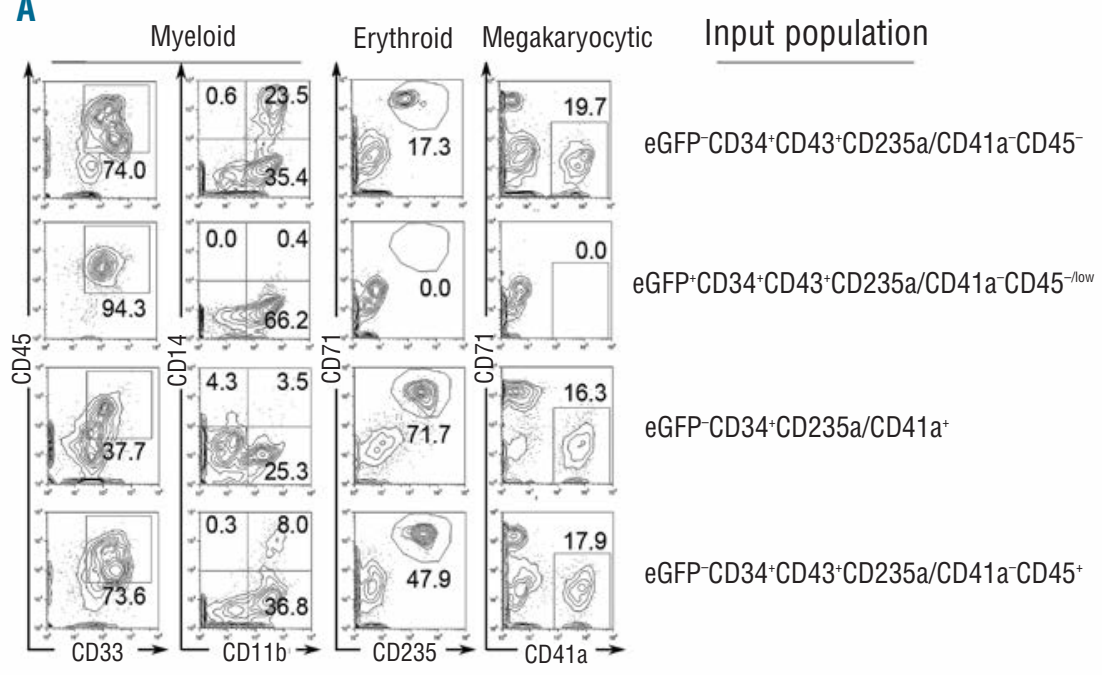

B
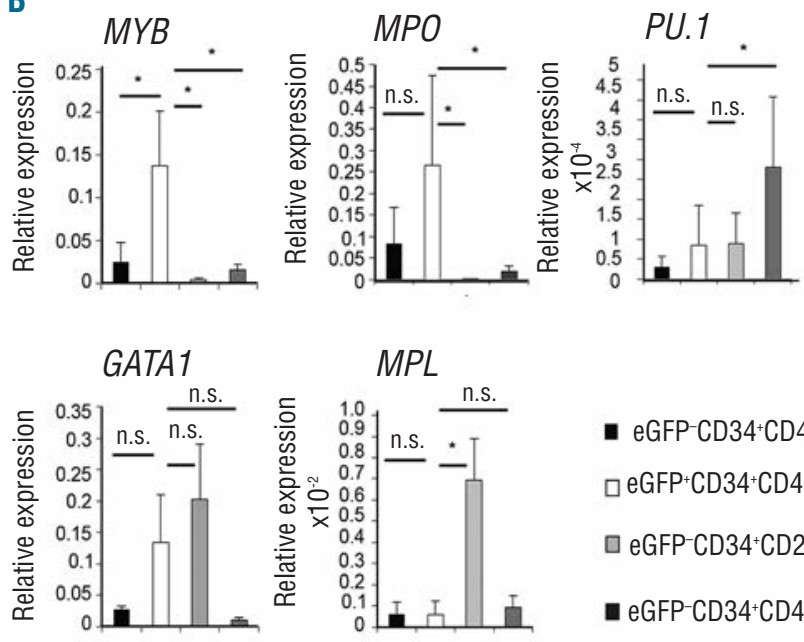

- eGFP-CD34+CD43+CD235a/CD41a-CD45口eGFP+CD34+CD43+CD235a/CD41a ${ }^{-} C D 45^{-110}$ 口 eGFP-CD34+CD235a/CD41a+

- eGFP-CD34+CD43+CD235a/CD41a-CD45+

Figure 5. MYB-eGFP ${ }^{+}$HPC show commitment towards the granulocytic lineage. (A) Flow cytometric analysis of differentiation cultures towards granulocytic, monocytic megakaryocytic and erythroid cells. Day 14 (d14) MYB-eGFP hESC-derived precursors with indicated phenotypes were isolated from IL-3 and FIt3-L expanded EB; representative plots of at least 3 independent experiments are shown. (B) qPCR analysis for lineage commitment genes on indicated populations, isolated from d14 MYB-eGFP hESC EB expanded in presence in IL-3 and Flt3-L. Expression is shown relative to the expression of GAPDH. Error bars indicate standard deviation (SD) of the mean $(n=3)$. were emerging directly from hemogenic endothelium, d11 $\mathrm{CD} 34^{+} \mathrm{CD}^{-} 3^{-}$endothelial cells were sorted and re-plated on OP9 stromal cells in medium containing SCF, BMP4 and VEGF. After six days of culture, a distinct CD45 population was apparent; however, no eGFP+ cells were seen at this time point. After 11 days of culture, $\mathrm{eGFP}^{+}$cells became apparent (Online Supplementary Figure S3). As this assay might miss the emergence of transient eGFP ${ }^{+}$cells directly from hemogenic endothelium, we used a similar set-up to analyze the emergence of hematopoietic cells from endothelium by live confocal imaging. During live confocal imaging, adherent eGFP-CD34+ CD $43^{-}$endothelial cells were found to round up and form cells with hematopoietic appearance. These cells subsequently upregulated CD43 and proliferated extensively over the course of $72 \mathrm{~h}$. We could not detect eGFP expression during the whole process of blood cell generation and subsequent proliferation, suggesting MYB-independent generation of HPC (Figure 3 and Online Supplementary Movie S1). Similar experiments initiated with sorted eGFP-CD34+ CD43- endothelial cells derived from earlier (d7) or later (d14) time points of EB cultures were much less potent in the generation of hematopoietic cells and generated only a few blood cells.

To verify whether other or additional growth factors were required for the generation and/or expansion of the $\mathrm{eGFP}^{+} \mathrm{CD} 4^{+} \mathrm{CD}_{3}{ }^{+} \mathrm{CD} 45^{-1 / 0}$ population, EB-derived hematopoietic cells were cultured in different cytokine combinations of SCF, TPO, Flt3-L, IL-3 and IL-6. It was found that eGFP ${ }^{+} \mathrm{CD} 34^{+} \mathrm{CD} 43^{+} \mathrm{CD}^{-1 / 10}$ cells expanded most efficiently in conditions containing IL-3. As shown in Figure $4 \mathrm{~A}$, the mean of absolute numbers of e $\mathrm{GFP}^{+} \mathrm{CD} 34^{+}$ $\mathrm{CD} 43^{+} \mathrm{CD} 45^{-1 / 0}$ per EB was approximately 6-fold higher than those in conditions without IL-3. This suggests that the eGFP ${ }^{+} \mathrm{CD} 34^{+} \mathrm{CD} 43^{+} \mathrm{CD} 45^{-1 / 0}$ cells are responsive to IL3 , as reported previously. ${ }^{11}$

\section{Characterization of eGFP negative and eGFP positive subsets}

To further define the eGFP+ cells generated in these cultures, we analyzed these cells for expression of the stem cell phenotype CD38-, CD90+, CD49f ${ }^{+}$and $\mathrm{CD}^{-} 4 \mathrm{RA}^{-}$as previously described by Notta et al., the myeloid lineage markers CD33 and CD123, and erythro-megakaryocytic lineage markers CD41a and CD235a. As shown in Figure $4 \mathrm{~B}$, eGFP ${ }^{+} \mathrm{CD} 34^{+} \mathrm{CD} 43^{+} \mathrm{CD} 45^{- \text {llo }}$ cells expressed low levels of CD45RA, were negative for CD38 and CD90, and expressed CD49f. This CD34 ${ }^{+}$CD 45RA ${ }^{-}$CD 38 ${ }^{-} \mathrm{CD}_{49} \mathrm{f}^{+}$ phenotype was present in all conditions, including the conditions containing IL-3, and is similar to the long-term 
engraftable HSC described by Notta et al. ${ }^{12}$ However, the $\mathrm{eGFP}^{+}$cells in our cultures also showed clear expression of CD33 and CD123, while erythroid lineage markers were negative, suggesting myeloid lineage commitment of these cells. The phenotype was very similar regardless of the cytokines added, except for the condition in EBmix + $\mathrm{TPO}+$ Flt3-L, where CD38 was up-regulated. CD235 and CD41a were consistently absent on these populations, arguing against erythroid commitment of eGFP ${ }^{+} \mathrm{CD} 34^{+} \mathrm{CD} 43^{+} \mathrm{CD} 45^{- \text {-1o }}$ cells.

The eGFP-CD $34^{+} \mathrm{CD} 43^{+} \mathrm{CD} 45^{-}$cells, on the other hand, contained mainly erythroid-committed cells, while the $\mathrm{CD} 45^{+}$population consisted of cells expressing myeloid lineage markers (data not shown). The e $\mathrm{GFP}^{+}$cells derived from eGFP- hemogenic endothelium, also phenotyped as $\mathrm{CD} 45^{\text {low }} \mathrm{CD} 33^{+} \mathrm{CD} 14^{-}$cells, consistent with the data obtained on bulk cultures (Online Supplementary Figure $S 3 B)$.

We assessed myeloid, erythroid and megakaryocytic differentiation capacity of the different populations obtained in the cultures with various growth factor mixes to assess multipotency. Results were qualitatively similar for all growth factor conditions. Figure 5A shows the results of sorted cell populations from d14 spin EB cultures expanded with EBmix plus IL-3 and Flt3-L, and subsequently assayed under conditions optimal for either myeloid, erythroid or megakaryocytic differentiation. The eGFP-CD34 ${ }^{+} \mathrm{CD}^{-} 3^{+} \mathrm{CD} 235 \mathrm{a} / \mathrm{CD} 41 \mathrm{a}^{-} \mathrm{CD} 45^{-}$population clearly contained progenitors giving rise to myeloid, erythroid and megakaryocytic cells, while the $\mathrm{eGFP}^{+} \mathrm{CD} 34^{+} \mathrm{CD} 43^{+} \mathrm{CD} 235 \mathrm{a} / \mathrm{CD} 41 \mathrm{a}^{-} \mathrm{CD} 45^{-/ \mathrm{l}}$ population was shown to give rise to granulocytic lineage myeloid cells only, with a complete absence of monocytic, erythroid and megakaryocytic precursor potential. On the other hand, eGFP-CD34 ${ }^{+} \mathrm{CD} 43^{+} \mathrm{CD} 235 \mathrm{a} / \mathrm{CD} 41 \mathrm{a}^{+}$cells gave rise mainly to erythroid and megakaryocytic cells, although some myeloid precursor activity was also observed. eGFP-CD $34^{+} \mathrm{CD} 43^{+} \mathrm{CD} 235 \mathrm{a} / \mathrm{CD} 41 \mathrm{a}^{-} \mathrm{CD} 45^{+}$cells were able to give rise to $\mathrm{CD} 11 \mathrm{~b}^{+} \mathrm{CD} 14^{-}$granulocytic lineage cells, $\mathrm{CD} 11 \mathrm{~b}^{+} \mathrm{CD} 14^{+}$macrophage lineage cells, $\mathrm{CD} 71^{+} \mathrm{CD} 235^{+}$erythroid and $\mathrm{CD} 41^{+}$megakaryocytic cells (Figure 5A).

To assess expression of genes associated or determining commitment towards the different lineages, RT-qPCR was performed for MPO, GATA1, PU.1 and MPL on the same sorted populations as in Figure 5A. MYB was assessed as a control and, as expected, showed high expression in the $\mathrm{eGFP}^{+}$population (Figure 5B). In line with the multipotent precursor capacity, the eGFP ${ }^{-} \mathrm{CD} 34^{+} \mathrm{CD} 43^{+} \mathrm{CD} 45^{-}$cells expressed only low levels of the various genes analyzed. In contrast, the eGFP- $\mathrm{CD} 34^{+} \mathrm{CD} 43^{+} \mathrm{CD} 45^{+}$cells had significantly higher levels of $P U .1$ suggesting that, together with the acquisition of CD45, the cells become committed to the macrophage lineage. As expected, the eGFP-CD $34^{+} \mathrm{CD} 235 \mathrm{a} / \mathrm{CD}^{-} 1 \mathrm{a}^{+}$express high levels of GATA1 and MPL, emphasizing their erythro-megakaryocytic commitment. In contrast, the eGFP ${ }^{+} \mathrm{CD} 34^{+} \mathrm{CD} 43^{+} \mathrm{CD} 45^{- \text {tho }}$ cells show high expression of $\mathrm{MPO}$ and low levels of PU.1 suggestive for granulocytic lineage commitment. GATA1 expression was also detected in the $\mathrm{eGFP}^{+} \mathrm{CD} 34^{+} \mathrm{CD} 43^{+} \mathrm{CD} 45^{- \text {-lo }}$ population, in agreement with granulocytic lineage commitment

To study the generation of the $\mathrm{eGFP}^{+}$precursor at the clonal level, CFU assays and single cell sorting followed
A

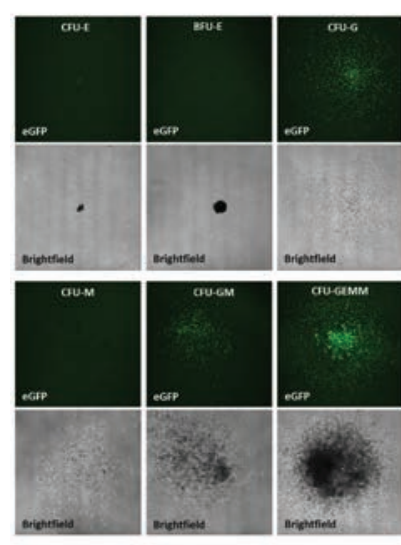

B

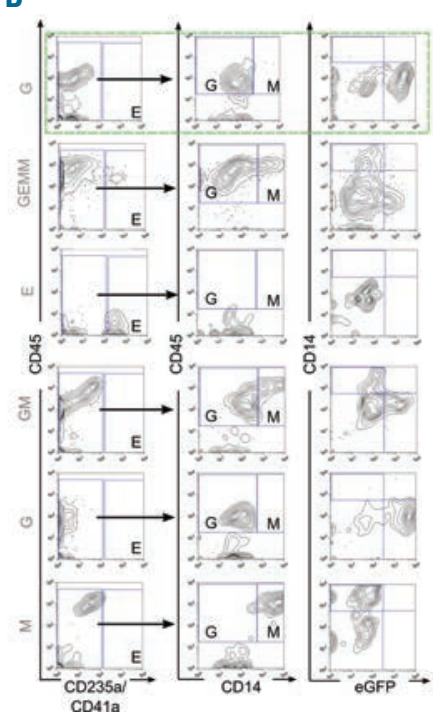

C
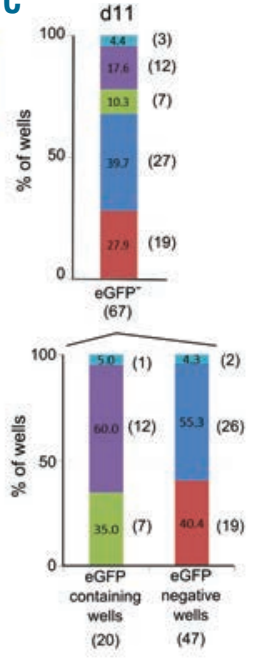
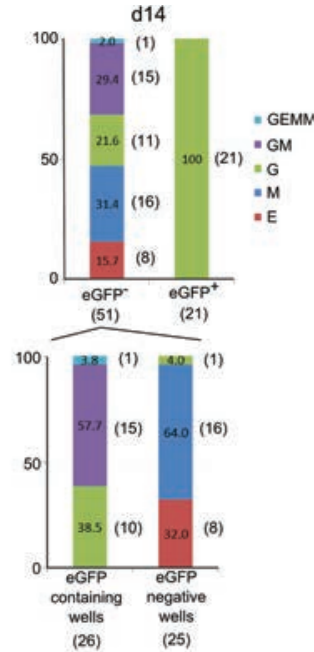

Figure 6. Clonal progeny analysis of $\mathrm{CD} 34^{+} \mathrm{CD} 43^{+}$progenitors. (A) Representative microscopic images of eGFP-CD34 ${ }^{+} \mathrm{CD} 43^{+} \mathrm{day} 11$ (d11) $^{-}$ embryoid bodies (EB) derived progenitor CFU assay, analyzed after 14 days of liquid culture. A representative image for each colony type is depicted. Both eGFP and brightfield channels are shown. (B) Representative analysis of single cell (clonal) cultured CD34 ${ }^{+}$CD43 ${ }^{+}$EB derived progenitors, analyzed after seven days of culture. CD235a/CD41a- cells are depicted in the center and right panels. Scoring gates are indicated with $\mathrm{E}$ (erythroid/megakaryocytic), $\mathbf{M}$ (monocytic) and $\mathrm{G}$ (granulocytic). Combination of these gates led us to determine colony type as $\mathrm{G}$ $\mathrm{M}, \mathrm{E}, \mathrm{GM}$ or GEMM as indicated for the representative plots. Representative progeny of eGFP+CD34 ${ }^{+} \mathrm{CD} 43^{+} \mathrm{EB}$ derived progenitors are depicted in the dotted box. (C) Frequency of progenitor types within CD34 $4^{+}$CD $43^{+}$EB derived progenitors at the indicated time points. Absolute numbers of colonies are indicated between brackets. The progeny of eGFP-CD34 ${ }^{+}$CD $43^{+}$are depicted in the panels below as fractions of wells containing eGFP $^{+}$cells or wells lacking eGFP ${ }^{+}$cells. At d14, progeny of the eGFP ${ }^{+} \mathrm{CD} 34^{+} \mathrm{CD} 43^{+}$population was assayed, next to the progeny of eGFP-CD $34^{+}{ }^{+} D 43^{+}$progenitors. All wells were scored as shown in (B). 
by liquid culture were performed on d11 and d14 EB cultures (Figure 6). The d11 eGFP- $\mathrm{CD} 34^{+} \mathrm{CD} 43^{+}$cell population gave rise to CFU-GEMM, CFU-GM, CFU-G, CFU$\mathrm{M}, \mathrm{BFU}-\mathrm{E}$ and CFU-E. These colonies were analyzed microscopically for eGFP expression and it was observed that the CFU-GM, CFU-G and CFU-GEMM contained eGFP positive cells whereas the CFU-M, CFU-E and BFUE were consistently negative (Figure 6A). Similarly, using single-cell culture and flow cytometric analysis, we found that $\mathrm{d} 11$ and $\mathrm{d} 14 \mathrm{eGFP}^{-} \mathrm{CD} 34^{+} \mathrm{CD} 43^{+}$progenitors consisted of GEMM, GM, G, M, E precursors. A marked skewing towards the myeloid lineage was observed on $\mathrm{d} 14$ of culture. In agreement with the CFU assays, eGFP ${ }^{+}$ cell containing wells were confined to the wells containing G, GM and GEMM precursor cells, whereas the wells lacking eGFP expression contained $\mathrm{E}$ and $\mathrm{M}$ precursors (Figure $6 \mathrm{~B}$ and $\mathrm{C}$ ). These data clearly show that the MYB-eGFP+ precursor is a more committed myeloid precursor derived from a multipotent MYB-eGFPhematopoietic precursor cell.
While macrophage development is MYB-independent, MYB marks granulocytic lineage differentiation

As MYB was described to be dispensable for the generation of YS-derived macrophages, we studied the role of MYB during myeloid lineage choice. When EB cultures were grown under myeloid differentiation conditions, a homogenous population of $\mathrm{CD}_{4} 5^{+} \mathrm{CD} 33^{+}$was obtained after four days of culture (Figure 7A). To further define both eGFP- and $\mathrm{eGFP}^{+}$populations, we analyzed both populations by flow cytometry, cytospin and qPCR.

The e $\mathrm{GFP}^{+}$population was found to be $\mathrm{CD} 45^{\text {lo }}$ and positive for CD33, weakly expressing the myeloid markers CD11c, CD11b, CD123 and CD13. The granulocytic marker CD15 was found absent. This phenotype is in line with the surface phenotype of myelocytes, a precursor of the granulocyte lineage. Macrophage markers CD14, CD16, CD115, HLA-DR and CD86 were consistently negative. On the other hand, the eGFP- population was found to be CD45 $5^{\mathrm{hi}}$ and $\mathrm{CD} 33^{+}$. These cells were

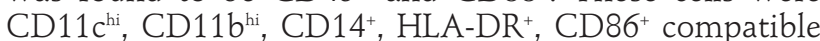

A

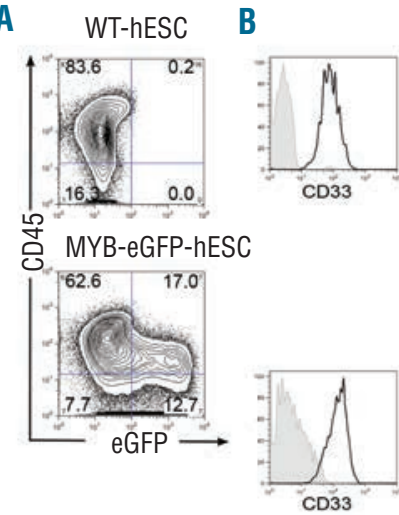

C

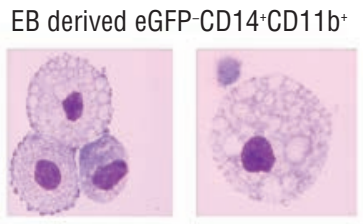

EB derived eGFP+CD14-

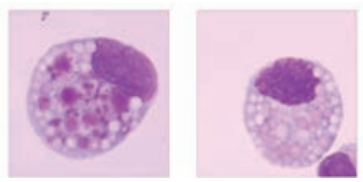

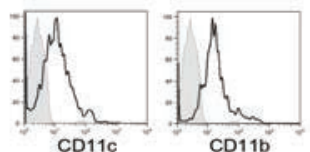
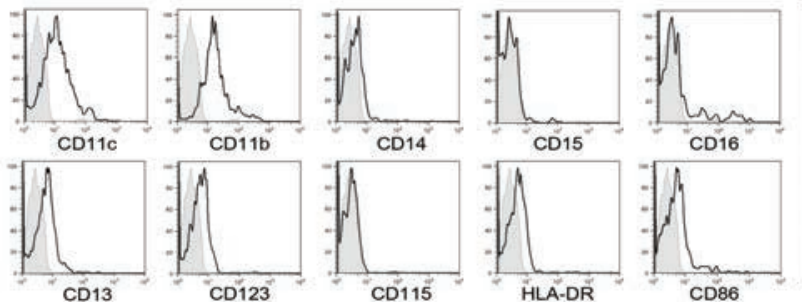

勆
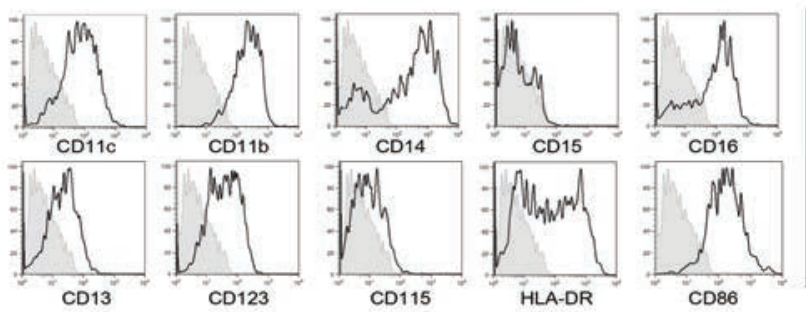

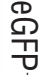

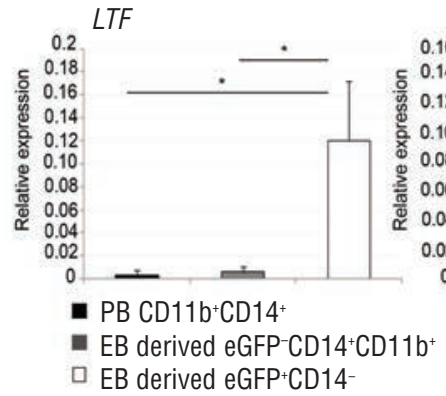

MYB

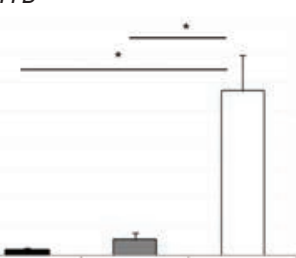

- EB derived eGFP-CD14+CD11 $\mathrm{b}^{+}$

Figure 7. Myeloid cells show strong expression of MYB-eGFP upon granulocytic lineage differentiation. (A) Flow cytometric analysis of myeloiddirected differentiation of day 11 MYB-eGFP hESC embryoid bodies, after 5 days of myeloid culture. Representative plots of at least 3 independent experiments are shown. (B) Phenotypic analysis of eGFP ${ }^{+}$(upper panels) and eGFP- (lower panels) populations in myeloid directed differentiation cultures of day 11 (d11) MYB-eGFP embryoid bodies after five days of myeloid culture. All cells shown in histograms are gated on CD45. Shaded histograms show control staining for the depicted marker. Representative plots of 3 independent experiments are shown. (C) May-Grünwald-Giemsa staining of cytospin samples of myeloid-directed differentiation of d11 MYB-eGFP hESC embryoid bodies, eGFP$\mathrm{CD}_{14}{ }^{+} \mathrm{CD} 11 \mathrm{~b}^{+}$and eGFP+CD14- populations are depicted. (D) qPCR analysis for lactoferrin (LTF) and MYB expression in indicated populations. Expression is shown relative to the mean of GAPDH and YWHAZ expression. Error bars indicate standard deviation (SD) of the mean ( $\mathrm{n}=2$ ). 
A

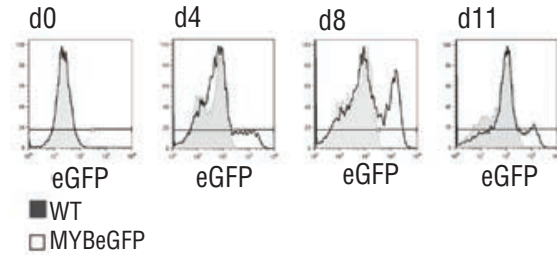

B
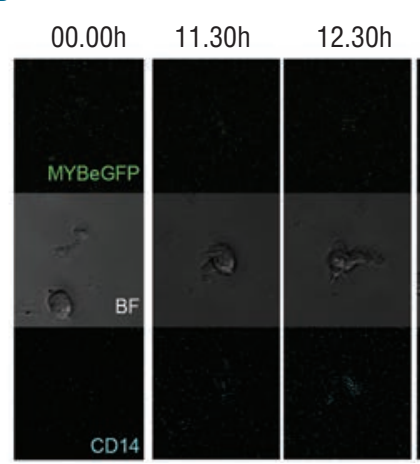

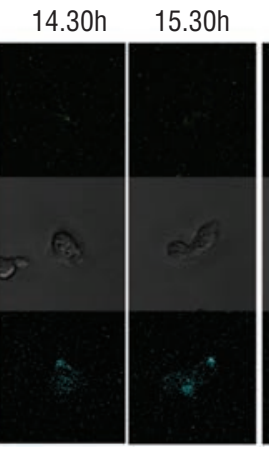

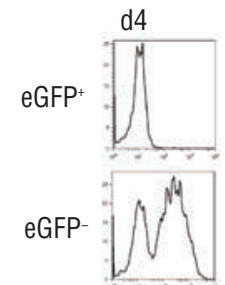

CD14

$16.30 \mathrm{~h}$

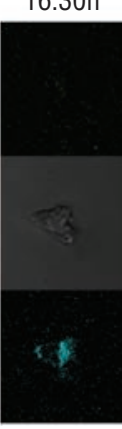

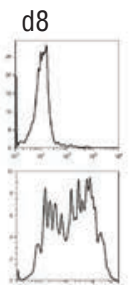

CD14

$17.30 \mathrm{~h}$

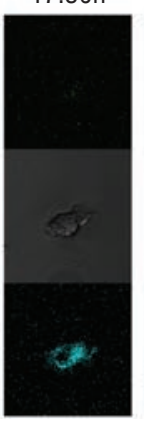

d11

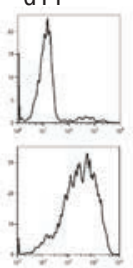

CD14

eGFP+ myeloid control

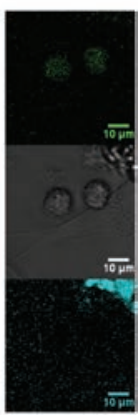

Figure 8. Macrophages develop without expression of MYB-eGFP and show characteristics of yolk sac-derived tissue macrophages. (A) Flow cytometric analysis of myeloid-directed differentiation of day 11 (d11) MYB-eGFP hESC embryoid bodies (EB)-derived eGFP-CD34 ${ }^{+} \mathrm{CD}^{-} 3^{+} \mathrm{CD}^{-} 5^{+}$ precursors, after 4,8 and 11 days of myeloid culture. (B) Time-lapse confocal analysis of live stained cultures. eGFP-CD $34^{+}{ }^{-} D 43^{+}$CD $45^{+}$CD $14^{-}$cells were cultured in myeloid differentiation conditions. Samples were live stained with CD14-PE for confocal analysis. eGFP ${ }^{+}$ myeloid cells are shown as reference. Single channel panels are shown every hour starting from $11.30 \mathrm{~h}$ after plating onwards. Scale bars measure $10 \mu \mathrm{M}$. with an activated macrophage phenotype. CD115, the receptor for M-CSF, was found only weakly positive. The cells also expressed CD16, a phenotype compatible with tissue macrophages (Figure 7B). On cytological analysis using May-Grünwald-Giemsa staining, eGFP ${ }^{+} \mathrm{CD} 14^{-}$cells show a granulocytic cytoplasm compatible with myelocytic granulocyte lineage cells, whereas the morphology of the eGFP-CD14+ cells is compatible with tissue macrophages (Figure 7C). This was confirmed by qPCR analysis showing these $\mathrm{eGFP}^{+}$cells to express the granule protein lactoferrin (LTF), eGFP- cells, on the other hand, were devoid of lactoferrin expression (Figure 7D). As expected, MYB expression levels were high in eGFP+CD14- cells (Figure 7D).

To analyze whether the generation of these macrophages from hematopoietic precursor cells was MYB-independent, we sorted eGFP-CD $34^{+} \mathrm{CD} 43^{+} \mathrm{CD} 45^{+}$ committed precursor cells, cultured these in myeloid conditions on OP9 stromal cells and analyzed them daily for the expression of eGFP. After four days, a population of CD14 ${ }^{+}$cells was already clearly visible. These cells were negative for eGFP as assessed by flow cytometry (Figure $8 \mathrm{~A})$. Further culture of these cells for up to 11 days showed clear eGFP positive populations at all analyzed time points. However, at no time point were $\mathrm{GFP}^{+} \mathrm{CD} 14^{+}$cells observed. After 11 days of culture, virtually all eGFP- cells were CD14 positive (Figure 8A).

To determine whether an eGFP ${ }^{+} \mathrm{CD} 14^{-}$intermediate stage gave rise to the eGFP-CD14+ macrophage cells, sorted $\mathrm{d} 11$ eGFP-CD $34^{+} \mathrm{CD} 43^{+} \mathrm{CD} 45^{+} \mathrm{CD} 14^{-}$committed HPC were cultured under myeloid conditions and analyzed using live confocal microscopy to track cells becoming CD14+. We did not observe eGFP expression in cells acquiring CD14 expression (Figure 8B and Online Supplementary Movie S2). These data suggest that the generation of $\mathrm{CD}_{14}{ }^{+}$ macrophage cells from hESC is MYB-independent.

\section{Discussion}

We here show evidence that multipotent HPCs expressing high levels of MYB are not generated in human EB cultures initiated with hESC. We, therefore, conclude that the hematopoietic cells generated in vitro from hESC, using current in vitro protocols, emerge through an endothelial intermediate, and are precursors with limited stem cell activity that resemble YS hematopoietic progenitors. In addition, we show that the first progenitors derived from endothelial cells are eGFP-CD $34^{+} \mathrm{CD} 43^{+}$cells, which then develop into $\mathrm{eGFP}^{+} \mathrm{CD} 34^{+} \mathrm{CD} 43^{+}$granulocyte committed progenitors.

Multipotency and self-renewal are two hallmark characteristics of HSC. With regard to multipotency, almost all hematopoietic cell types can be generated from hESCderived hematopoietic precursors, including erythrocytes,${ }^{13}$ megakaryocytes,${ }^{14}$ granulocytes, monocytes,${ }^{15} \mathrm{NK}$ cells ${ }^{16}$ and T cells. ${ }^{17,18}$ Although, to our knowledge, it has not been demonstrated that a single hESC-derived hematopoietic precursor cell is able to form all of the aforementioned cell types, the absence of these reports may be due to technical issues associated with precursor cells of limited proliferative capacity. The fact that T cells can be generated from fetal HPC does not in itself prove the presence of multipotent progenitors or HSC, since it has been shown that T cells can be generated from YS precursors that arise before HSC are generated. ${ }^{19,20}$ Selfrenewal of HSC is evaluated by their ability to reconstitute immune deficient mice. Previous studies have reported re-population by in vitro hESC-derived HPC. ${ }^{21,22}$ Wang et al. reported multilineage hematopoietic repopulation upon intrafemoral injection of HPC. ${ }^{22}$ However, hESCderived HPCs had limited proliferative and migratory capacity compared with somatic HSCs. In another study by Ledran et al., whole hESC differentiation co-cultures 
with stromal cells derived from murine fetal tissues were injected intrafemorally in mice. These cells were reported to re-populate bone marrow of the non-injected femur as well as the spleen. In addition, secondary engraftment was reported. However, the engrafted cells were not fully defined. The phenotype shown is compatible with myeloid cells $\left(\mathrm{CD} 33^{+} \mathrm{CD} 13^{+}\right)$. As it is known that tissue macrophages have a long lifespan, and are found lifelong in the adult, without a need for HSC to replenish them, chimerism may have been caused by cells that are not HSC dependent. There was no evidence for engraftment of $\mathrm{CD} 34^{+}$cells in the bone marrow.

In a recent article by Amabile et al., hESC were injected in mice to form teratoma. The authors show that in these teratoma, also hESC derived $\mathrm{CD}^{+} 4^{+}$hematopoietic cells are formed. Upon isolation and transplantation of these $\mathrm{CD}^{+} 4^{+} \mathrm{HPC}$ into immunodeficient mice, engraftment of human cells was found, including $\mathrm{CD}_{3} 4^{+}$cells in the bone marrow. This in vivo differentiation model, therefore, suggests that HSC can be generated from human pluripotent stem cells. $^{23}$

In conclusion, based on these data, it remains questionable whether HSC can be formed from ESC using currently available in vitro differentiation protocols, although it is clear that multipotent progenitors are formed. Changes to the culture conditions, such as other cytokine mixtures or the use of more appropriate feeder lines may result in HSC generation. The MYB-eGFP cell line that we generated will be very helpful in screening for such conditions. We still do not know which factor(s) needed to generate HSC is still missing. However, recent publications using reprogramming of specified cells are starting to shed light on this issue. ${ }^{24-26}$ It was recently reported that overexpression of 5 factors in hESC-derived HPC confers short-term engraftment potential to these hESC-derived cells. ${ }^{24}$ One of the factors which was required for in vivo engraftment was characterized as MYB. A similar strategy was used for reprogramming murine hematopoietic cells toward transplantable HSC. ${ }^{26}$ Although the reprogramming factors needed for conferring HSC properties have not yet reached consensus, this approach may prove to be an alternative method for generating HSC from pluripotent stem cells.

An alternative hypothesis could be that HSC are continuously generated in vitro, but lack necessary signals that are present in vivo, and thus quickly degenerate to restricted precursor cells, losing their self-renewal capacity. Fetal, newborn and adult HSC are known to lose the capacity to self-renew within days of in vitro culture. ${ }^{27-29}$

To study the emerging HPC in vitro, we isolated CD34+ CD43- endothelial cells and studied the characteristics of hematopoietic cells generated from them through timelapse microscopy. We did not observe generation of MYBeGFP expressing cells that subsequently lose MYB expression in vitro. This is in line with the reported generation of hematopoietic cells directly from hemogenic endothelium from $M y b^{-1-}$ murine ESC, showing MYB to be dispensable for endothelial to hematopoietic transition. ${ }^{30}$ Instead, we observed either cells that remained MYB negative, or cells, which up-regulated MYB over the course of several days and were further characterized as granulocyte lineage cells. In the murine system, strong evidence was provided that MYB is essential for HSC function. Transplantable HSC were shown to express high levels of $M y b{ }^{6}{ }^{6}$ This is strengthened by the fact that $M y b^{-1}$ mice die around fetal d15 due to lack of transplantable HSC; ${ }^{4}$ moreover, conditional deletion of Myb leads to exhaustion of the stem cell pool and failure to engraft upon transplantation of LSK cells. ${ }^{6}$ We can, therefore, conclude that it is unlikely that HSC are generated in these spin EB cultures.

Conditional MYB knockout models show a marked decrease in granulocytic development, suggesting an important role for MYB in granulocytic development. ${ }^{7}$ In addition, hematopoietic differentiation cultures set up with $M y b^{-1}$ murine ESC also showed defective granulocyte lineage differentiation. ${ }^{30,31}$ Clarke et al. reported a similar potential to generate CFU-E and CFU-M between wild-type and $M y b^{-1}$ mESC in short-term cultures; however, later on, the numbers of CFU-E and CFU-GM were decreased. Sakamoto et al. compared wild-type with $M y b^{-1}$ mES cells and knock out mES cells that expressed Myb under control of a tetracyclin-inducible promotor. They reported similar numbers of hematopoietic cells generated by wild-type and knock-out cells, whereas induction of $M y b$ during hematopoietic differentiation resulted in vastly higher cell numbers. Similar to Clarke et al., they report a reduced differentiation towards erythroid and granulocytic lineages, whereas the monocytic differentiation is unaffected. The relative absence of these lineages in mESC cultures reflects the MYB dependency of a lineage restricted erythroid-megakaryocytic and erythro-myeloid precursor rather than the generation of MYB-dependent HSC. These data thus point towards a differential requirement of MYB during macrophage/granulocyte commitment, as is also apparent in our experiments.

Recent studies suggest that tissue macrophages belong to a separate lineage derived from MYB-independent precursors. These are derived from YS-derived EMP and persist by local proliferation of terminally differentiated cells or precursors into adult life independent from the bone marrow. The MYB-eGFP- macrophage cells generated in our cultures, co-express CD14 and CD16, a phenotype previously described for tissue macrophages. ${ }^{32,33}$

In conclusion, we have shown that hematopoietic precursor cells that arise from endothelial cells in EB cultures are MYB negative. MYB-positive precursors arise later in the cultures and are granulocyte lineage restricted. These data, therefore, provide evidence that bona fide HSC are neither generated nor maintained in these cultures. Rather, yolk sac-like hematopoietic precursors are formed. We here describe that granulocytic lineage committed progenitor diverge thereof, and that this process is accompanied by the upregulation of $M Y B$ expression. In this light, reinvestigation of $M y b$ signal in YS precursors might be advised. In addition, we have described a $M Y B$ reporter cell line, which may be helpful to screen for conditions that can generate HSC from hemogenic endothelium in vitro.

\section{Acknowledgments}

The authors would like to thank Wendy Toussaint for help with BAC modification, Sophie Vermaut for help with flow cytometry and cell sorting, Carine De Buck and Sabrina Seghers for performance and analysis of CFU assays.

\section{Funding}

This work was supported by the Research Foundation Flanders (Fonds voor Wetenschappelijk Onderzoek Vlaanderen, FWO), Stichting tegen Kanker, the "Interuniversity Attraction Poles" (IAP) Program of the Belgian Science Policy Office and the 
Geconcerteerde Onderzoeksactiviteiten (GOA) of Ghent University. SV, YVC and GV are supported by the Instituut voor de Aanmoediging van Innovatie door Wetenschap en Technologie in Vlaanderen (IWT).

\section{Authorship and Disclosures}

Information on authorship, contributions, and financial \& other disclosures was provided by the authors and is available with the online version of this article at www. haematologica.org

\section{References}

1. Medvinsky A, Dzierzak E. Definitive hematopoiesis is autonomously initiated by the AGM region. Cell. 1996;86(6):897-906.

2. Palis J, Robertson S, Kennedy M, Wall C, Keller G. Development of erythroid and myeloid progenitors in the yolk sac and embryo proper of the mouse. Development. 1999;126(22):5073-5084

3. Tober J, McGrath KE, Palis J. Primitive erythropoiesis and megakaryopoiesis in the yolk sac are independent of c-myb. Blood. 2008;111(5):2636-2639.

4. Mucenski ML, McLain K, Kier AB, et al. A functional c-myb gene is required for normal murine fetal hepatic hematopoiesis. Cell. 1991:65(4):677-689.

5. Bertrand JY, Chi NC, Santoso B, Teng S, Stainier DY, Traver D. Haematopoietic stem cells derive directly from aortic endothelium during development. Nature. 2010;464(7285):108-111.

6. Lieu YK, Reddy EP. Conditional c-myb knockout in adult hematopoietic stem cells leads to loss of self-renewal due to impaired proliferation and accelerated differentiation. Proc Natl Acad Sci USA. 2009;106(51): 21689-21694.

7. Lieu YK, Reddy EP. Impaired adult myeloid progenitor CMP and GMP cell function in conditional c-myb-knockout mice. Cell Cycle. 2012;11(18):3504-3512.

8. Schulz C, Gomez Perdiguero E, Chorro L, et al. A lineage of myeloid cells independent of $\mathrm{Myb}$ and hematopoietic stem cells. Science. 2012;336(6077):86-90.

9. Kyba M, Perlingeiro RC, Daley GQ. HoxB4 confers definitive lymphoid-myeloid engraftment potential on embryonic stem cell and yolk sac hematopoietic progenitors. Cell. 2002;109(1):29-37.

10. Ng ES, Davis RP, Azzola L, Stanley EG, Elefanty AG. Forced aggregation of defined numbers of human embryonic stem cells into embryoid bodies fosters robust, reproducible hematopoietic differentiation. Blood. 2005;106(5):1601-1603.

11. Vodyanik MA, Thomson JA, Slukvin, II. Leukosialin (CD43) defines hematopoietic progenitors in human embryonic stem cell differentiation cultures. Blood. 2006; 108(6):2095-2105.

12. Notta F, Doulatov S, Laurenti E, Poeppl A,
Jurisica I, Dick JE. Isolation of single human hematopoietic stem cells capable of longterm multilineage engraftment. Science. 2011;333(6039):218-221.

13. Qiu C, Olivier EN, Velho M, Bouhassira EE Globin switches in yolk sac-like primitive and fetal-like definitive red blood cells produced from human embryonic stem cells. Blood. 2008;111(4):2400-2408.

14. Gaur M, Kamata T, Wang S, Moran B, Shattil SJ, Leavitt AD. Megakaryocytes derived from human embryonic stem cells: a genetically tractable system to study megakaryocytopoiesis and integrin function. J Thromb Haemost. 2006;4(2): 436-442.

15. Choi KD, Vodyanik MA, Slukvin, II. Generation of mature human myelomonocytic cells through expansion and differentiation of pluripotent stem cell-derived linCD34+CD43+CD45+ progenitors. J Clin Invest. 2009;119(9):2818-2829.

16. Woll PS, Martin CH, Miller JS, Kaufman DS. Human embryonic stem cell-derived NK cells acquire functional receptors and cytolytic activity. J Immunol. 2005 175(8):5095-5103.

17. Timmermans F, Velghe I, Vanwalleghem I et al. Generation of $\mathrm{T}$ cells from human embryonic stem cell-derived hematopoietic zones. J Immunol. 2009;182(11):6879-6888.

18. Kennedy M, Awong G, Sturgeon CM, et al. $T$ lymphocyte potential marks the emergence of definitive hematopoietic progenitors in human pluripotent stem cell differentiation cultures. Cell Rep. 2012; 2(6):17221735.

19. Yoshimoto M, Porayette P, Glosson NL, et al. Autonomous murine T-cell progenitor production in the extra-embryonic yolk sac before HSC emergence. Blood. 2012; 119(24):5706-5714.

20. Ramond C, Berthault C, Burlen-Defranoux $O$, et al. Two waves of distinct hematopoietic progenitor cells colonize the fetal thymus. Nat Immunol. 2014;15(1):27-35.

21. Ledran MH, Krassowska A, Armstrong L, et al. Efficient hematopoietic differentiation of human embryonic stem cells on stromal cells derived from hematopoietic niches. Cell Stem Cell. 2008;3(1):85-98.

22. Wang L, Menendez P, Shojaei F, et al Generation of hematopoietic repopulating cells from human embryonic stem cells independent of ectopic HOXB4 expression. J Exp
Med. 2005;201(10):1603-1614

23. Amabile G, Welner RS, Nombela-Arrieta C et al. In vivo generation of transplantable human hematopoietic cells from induced pluripotent stem cells. Blood. 2013; 121(8):1255-1264

24. Doulatov S, Vo LT, Chou SS, et al. Induction of multipotential hematopoietic progenitors from human pluripotent stem cells via respecification of lineage-restricted precursors. Cell Stem Cell. 2013;13(4):459-470

25. Pereira CF, Chang $B$, et al. Induction of a hemogenic program in mouse fibroblasts. Cell Stem Cell. 2013;13(2):205-218.

26. Riddell J, Gazit R, Garrison BS, et al Reprogramming committed murine blood cells to induced hematopoietic stem cells with defined factors. Cell. 2014;157(3):549 564

27. Rollini P, Kaiser S, Faes-van't Hull E, Kapp U Leyvraz S. Long-term expansion of transplantable human fetal liver hematopoietic stem cells. Blood. 2004; 103(3):1166-1170.

28. Huang J, Nguyen-McCarty M, Hexner EO Danet-Desnoyers G, Klein PS. Maintenance of hematopoietic stem cells through regulation of Wnt and mTOR pathways. Nat Med 2012;18(12):1778-1785

29. Conneally E, Cashman J, Petzer A, Eaves C Expansion in vitro of transplantable human cord blood stem cells demonstrated using a quantitative assay of their lympho-myeloid repopulating activity in nonobese diabeticscid/scid mice. Proc Natl Acad Sci USA. 1997;94(18):9836-9841.

30. Sakamoto H, Dai G, Tsujino K, et al. Proper levels of c-Myb are discretely defined at distinct steps of hematopoietic cell development. Blood. 2006;108(3):896-903.

31. Clarke D, Vegiopoulos A, Crawford A, Mucenski M, Bonifer C, Frampton J. In vitro differentiation of c-myb(-/-) ES cells reveals that the colony forming capacity of unilineage macrophage precursors and myeloid progenitor commitment are cMyb independent. Oncogene. 2000; 19(30):3343-3351

32. Ziegler-Heitbrock HW, Fingerle G, Strobel $M$, et al. The novel subset of CD14+/CD16+ blood monocytes exhibits features of tissue macrophages. Eur J Immunol. 1993;23(9): 2053-2058.

33. Gordon S, Taylor PR. Monocyte and macrophage heterogeneity. Nat Rev Immunol. 2005;5(12):953-964. 